\title{
Transforaminal block of the dorsal ganglion of the root of L5, transfacetary approach: description of an alternative approach
}

\begin{abstract}
Lumbosciatica is considered one of the most prevalent types of pain. It is also among the most resource-intensive conditions to treat with conservative, invasive, and surgical treatments. Lumbar pain with mechanical characteristics is one of the predominant clinical features of lumbar spondylarthrosis, although it can also cause stenosis of the lumbar spinal canal due to the degenerative changes that occur and the obliteration of the foramina, producing a predominantly radicular pain.

Corticoid infiltration of the epidural space can be used to treat and manage radicular pain produced by any of the aforementioned causes. One approach to this procedure is the transforaminal approach. This paper presents an alternative to the classical intra-articular approach when there are challenges present that may complicate the performance of the technique using the previously described classical approaches.
\end{abstract}

Volume 3 Issue 3 - 2018

\author{
Abejón D,' Zamora A, ${ }^{2}$ Arango S, ${ }^{2}$ Monzón, \\ E,' Horas $C^{2}$, Márquez $\mathrm{G}^{2}$ \\ 'Department Head for the Pain Management Unit, Quiron Salud \\ Hospital Group, Spain \\ 2Pain Management Unit, Quiron Salud Hospital Group, Spain
}

Correspondence: David Abejón, Department Head for the Pain Management Unit, Universidad Europea de Madrid, Hospital Universitario Quiron Salud Madrid,Spain, Tel +3464922409I, Email dabejongonzalez@gmail.com

Received: May 01, 2018| Published: May 07, 2018

\section{Introduction}

In a recent report, the American Institute of Medicine (IoM) demonstrated that chronic pain affects about 100 million adults, at an approximate cost of $\$ 635$ billion per year including medical costs and lost productivity. ${ }^{1}$ Lumbosciatica is considered one of the most prevalent types of pain, as well as being one of the most resourceintensive conditions to treat using conservative, invasive, and surgical treatments. ${ }^{2}$ The main causes that may contribute to this syndrome include lumbar surgery or other conditions brought on by other causes, such as a herniated disc, degenerative disease, spondylarthrosis, tumors, or nerve compression.

This type of pathology must be treated using a multi- and interdisciplinary approach in order to develop a treatment that addresses all aspects of the pathology, including the affective and sensory aspects, ${ }^{3}$ which may involve measures such as taking prescription drugs (if needed), physical therapy ${ }^{4}$ and interventional approaches. $^{5}$

There are multiple reviews that discuss the use of pharmacological drugs for the treatment of neuropathic pain. These reviews mention various drugs that can be used for this type of pathology, ${ }^{6}$ although there is a significant percentage of ${ }^{7}$ patients who do not respond adequately to this type of treatment; in such cases, an interventional approach is needed to achieve better outcomes.

A predominant clinical feature of lumbar spondylarthrosis is lumbar pain with mechanical characteristics caused by the deformity and alteration of the interapophyseal joints, although it can also cause stenosis of the lumbar spinal canal due to the degenerative changes that occur, and it can cause obliteration of the foramina due to lumbar vertebral deformities ${ }^{8}$ and the thickening of the yellow ligament; the zygapophyseal joints and surrounding tissues ${ }^{9}$ produce a predominantly radicular pain.

Corticoid infiltration of the epidural space have been widely used to treat and manage radicular pain produced by any of the aforementioned causes due to the anti-inflammatory action of the steroids, which inhibit the synthesis of prostaglandins and block the nerve conduction of C-fibers, thus controlling perineural edema. ${ }^{10,11}$ Among the variations of this type of infiltration, the transforaminal approach seems to be more appropriate than the caudal or interlaminar approach because the concentration of the drug is highest near the affected nerves, and because this approach allows the drug to be placed in the anterior epidural space of the nerve. ${ }^{12}$

This paper presents a case study in which the difficulties presented by the classical approach force us to select alternative approaches, accounting for the previous anatomical study.

\section{Clinical case}

The subject of this case study is a 65 -year-old woman with no relevant medical history or drug allergies. Her surgical history included an arthroscopic knee surgery due to meniscopathy, as well as a previous lumbar spine surgery (decompression without arthrodesis/ instrumentation). She was admitted to the Pain Management Unit, presenting with lumbar pain that radiated throughout the lower left limb, including the inguinal and perineal regions. The symptoms had been evolving for multiple years with moderate-severe intensity. Upon examination, a normal pain threshold was observed, with mobility limited by pain. Pain was present at the midline and in the left facet midline, as well as pain at the insertion point of the iliolumbar ligament and the thoracolumbar fascia at its insertion point in the sacroiliac joint. The lumbar and paravertebral musculature were not examined at this time. The neurological examination revealed a pathological Lasegue and Braggard test at $45^{\circ}$, with normal reflexes, sensitivity, and motor function examination. Regarding supplementary tests, she underwent a magnetic resonance imaging (MRI) test that provided the following findings: degenerative scoliosis with right lateral displacement of L3, moderate-severe osteodegenerative changes in posterior elements and osteochondrosis in the L1-L4 segment, L3-L4 protrusion in the inferior and extraforaminal recess of the left side, L4-L5 disc protrusion, and L5-S1 disc degeneration. Medical treatment with 
opioids/neuromodulators (e.g. gabapentin, tricyclic antidepressants) was initiated, and various interventional techniques were performed, including a caudal epidurolysis and bilateral sacroiliac joint blocks, but the various techniques failed and no positive outcomes were achieved. Given the severity of the condition and the failures of previous treatments, a transforaminal block was performed on the left dorsal root ganglion (DRG) of L2, L3 and L4, with the intent to treat these ganglia using pulsed radiofrequency if the results were positive.

\section{Technique}

A transforaminal (TF) block was proposed at L2, L3, and L4 on the left side. After explaining the technique and its possible complications, which include the risks of infection, bleeding, and allergic reactions, oral and written consent was obtained, and the nature of the technique was explained to the patient, i.e. to perform a diagnostic block to determine the source of her pain. The technique was performed in the operating room on an outpatient basis, with standard monitoring according to SEDAR [Spanish Society of Anesthesia, Recovery, and Pain Therapy] standards (including EKG, O2 saturation, and non-invasive blood pressure tests) and radiological monitoring. The interventionist located the treatment sites with the patient lying in the prone position, using an abdominal pillow to reduce lumbar lordosis. During the procedure, a Yale $22 \mathrm{G}$ spinal needle was used for all treatment sites. The area was cleaned with chlorhexidine solution and misted so that the surgical field was completely sterile, with the area to be treated left exposed. A TF block was performed at the level of L2 without difficulty, although the TF block at the level of L3 was very difficult due to the patient's anatomy, which presented with a lateral displacement of L3 and abundant osteophytes on the vertebral body, making it very difficult to visualize the entry point through the foramen. For this reason, it was deemed more appropriate to enter through the L3-L4 facet joint. In order to perform the block at this location, the $\mathrm{C}$-arm of the fluoroscope was tilted obliquely until the spinous process was brought to the contralateral pedicle axis, or until the superior articular process could be seen in the middle part of the intervertebral disc, forming the famous "Scottie dog" sign, at an oblique inclination of approximately $20-30^{\circ}$; the double contour of the intervertebral disc was corrected with a craniocaudal inclination of the $\mathrm{C}$-arm. After this maneuver, the $\mathrm{X}$-ray was inclined craniocaudally $\left(20^{\circ}\right)$ to separate the iliac crest and allow entry into the foramen, according to the usual technique. ${ }^{13}$ Given the impossibility of visualizing the entry point, the $\mathrm{C}$-arm was forced to incline in both directions (obliquely and craniocaudally), without achieving visualization (Figure 1). As seen in Figure 2 the only entry point that allowed access to the desired target was through the zygapophyseal joint. To perform this approach, this joint was centered and the $\mathrm{C}$-arm was rotated until the facet joint could be visualized correctly. The target for this type of approach is the middle part of the joint. The approach was done in "tunnel vision" (the needle was positioned with its axis parallel to the X-rays being emitted), advancing along this direction until it reached the location of the L3 DRG. The depth of the needle was checked using a lateral $\mathrm{X}$-ray view (Figure 3). The needle was pushed slightly deeper through the facet joint until it reached the epidural space and the dorsal root ganglion of L3. Entry into the joint is perceived as a loss of resistance as the needle penetrates the synovial membrane. Once it entered into the joint, the location was verified by injecting 0.1 to $0.3 \mathrm{~mL}$ of contrast medium (Figure 4 ). After verifying that the needle was placed perfectly, local anesthetics and corticosteroids could be injected. In this case, the interventionist used $0.25 \%$ bupivacaine and triamcinolone, in an amount not exceeding 1-2 mL.

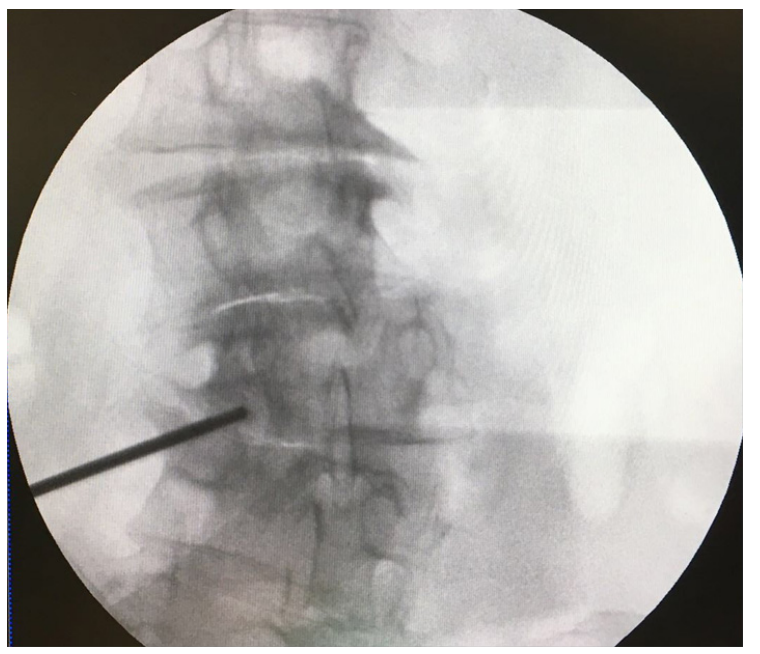

Figure I *Target at the entry point for the L2 and L4 blocks ** Entry point into the zygapophyseal joint at the level of $L 3$ due to the impossibility of using the entry point for the classic subpedicular approach.

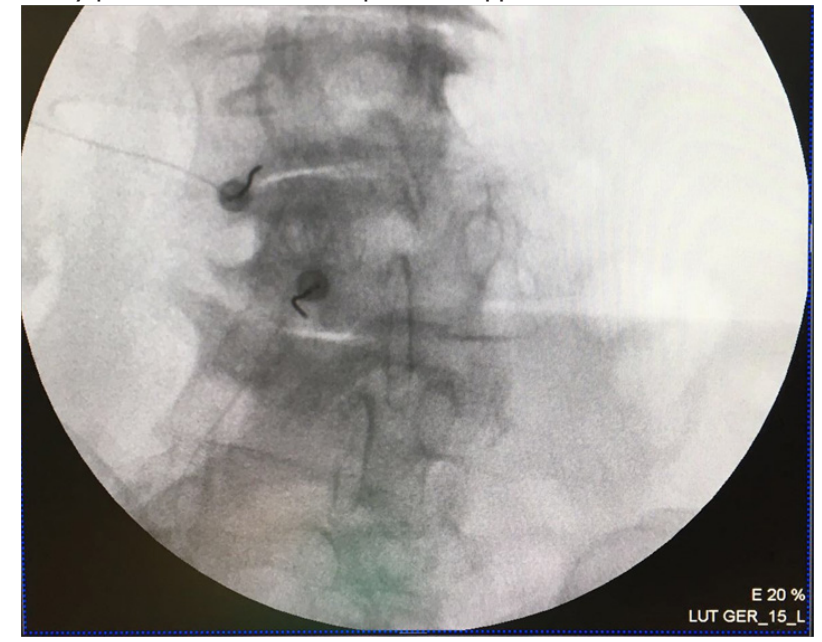

Figure 2 I Entry of the needle into the zygapophyseal joint for L3.

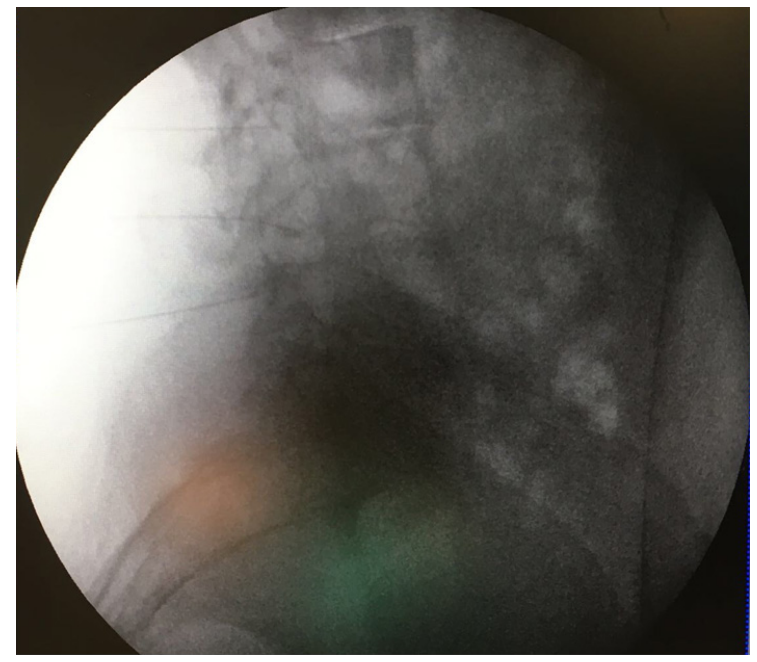

Figure 3 Lateral view of the transforaminal block. See the entrance through the joint into the L3 space (I). 


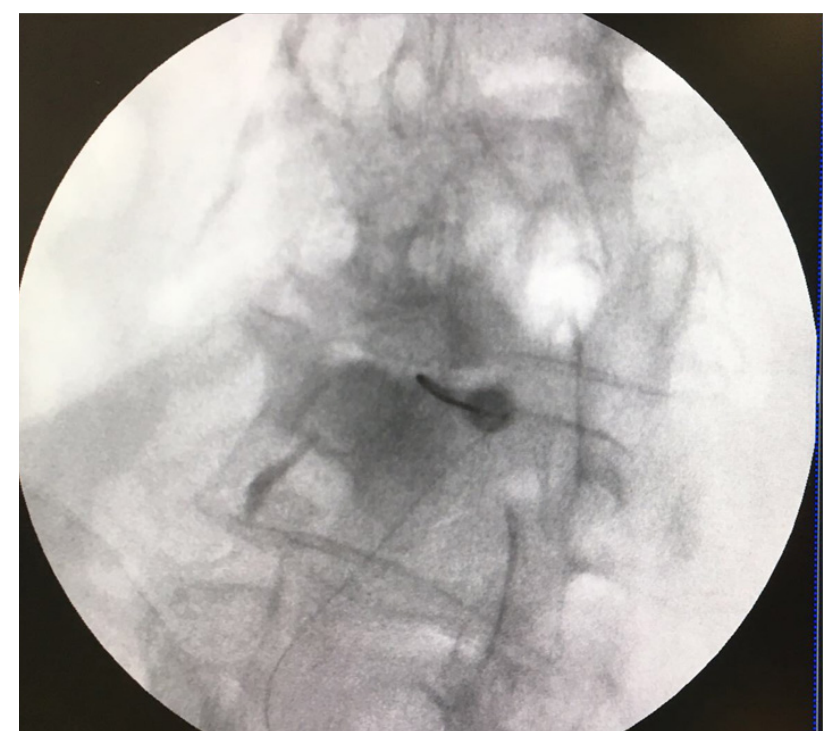

Figure 4 Contrast is used to visualize the location where the root exits (I) with the needle in the interapophyseal joint (2).

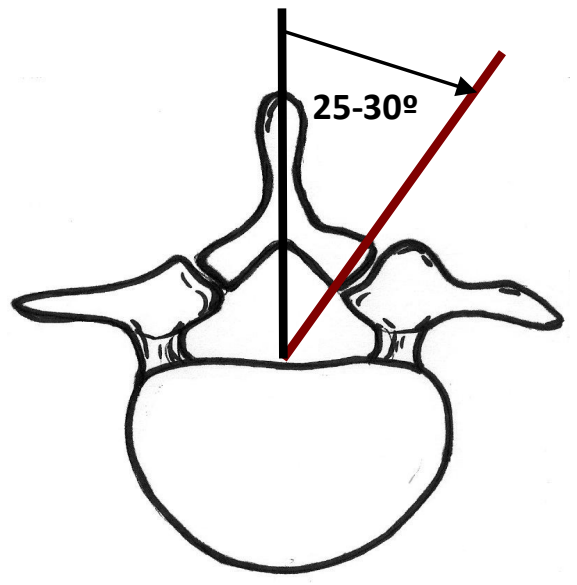

Figure 5 Cephalic view of lumbar vertebra. The facet angulation is indicated.

The patient was discharged with no complications arising from the technique. At the 4-week follow-up consultation, the patient reported $>50 \%$ relief of pain with no complications.

\section{Discussion}

Initial non-surgical treatment of patients with radiculopathy begins with conservative measures: medical treatments (e.g. neuroleptics, opioids, and antidepressants $)^{14}$ and physical therapy. When these do not work, the treatment plans that follow usually consist of interventional measures, which include: epidural injection of local anesthetics and corticosteroids, selective root block, ${ }^{15}$ pulsed radiofrequency of the DRG, ${ }^{16}$ epidurolysis, ${ }^{17}$ and if all else fails, neurostimulation. ${ }^{18}$ These techniques are not without risk, but they may be necessary if conservative measures are not effective. For each technique, the level of evidence attesting to their effectiveness at controlling radicular pain is inconclusive, ${ }^{19}$ but they seem to be effective in the short- to medium-term; $\mathrm{PRF}^{20}$ and epidurolysis techniques have the longest duration. ${ }^{21}$ Because the pain is neuropathic in nature, ablative techniques are contraindicated.
The patient presented in this paper received medical treatment, but the results were not satisfactory. Therefore, it is recommended that she receive a selective DRG block for subsequent PRF technique.The lumbar DRG is located in the cranial part of the intervertebral foramen, just below the pedicle. In the upper regions of the lower back, their locations are more posterior than their corresponding locations in the lower regions (such as in L4 and L5) because of their relationships with the anterior rami. The roots of the upper levels assume a more posterior and medial direction than those of the lower levels, as seen in the present case with the position of the root of L3. ${ }^{13}$

The patient suffers from spondylarthrosis, so after placing the radiological C-arm to perform the usual approach (ipsilateral oblique and slight caudocranial inclination), the patient's anatomy presented with significant lateral displacement and the formation of osteophytes ( $1 \mathrm{~mm}$ below the pedicle) while attempting the so-called "classical approach". It was impossible to adequately visualize the entry point or find an opening that was adequate for entry into the foramen without risking permanent nerve damage. There are several approaches for this type of procedure, including the Kambin's triangle approach and the subpedicular approach. The Kambin triangle ${ }^{22}$ is a triangular shape formed by the dorsolateral disc. The hypotenuse is the emerging nerve, the base is the upper edge of the lower vertebra, and the height is perpendicular to the nerve root. With this idea in mind, the ganglion can be approached from various directions, such as the retrodiscal and the preganglionic approaches. The concept of the spinal nerve canal is also derived from this idea, defined as the space occupied by the nerve from the point where it becomes visible until it leaves the intervertebral foramen, divided into three parts: the canal at the entry point, the middle part, and the exit location.

The intervertebral foramen is formed by the union of the various osseous elements of each vertebra. The anterior portion contains the vertebral bodies and intervertebral discs (anterior compartment), laterally delimited by the junction where the pedicles meet and posteriorly delimited by the laminae. The facet joint is formed by the junction where the superior articular process of the inferior vertebra meets with the inferior articular process of the superior vertebra. The lateral angulation of each vertebra at each level allows us to visualize the ranges of movement. As seen in Figure 5, in the transverse plane of the lumbar facet joint, there is a lateral angulation of about 25$30^{\circ}$. In this manner, if we position the $\mathrm{C}$-arm of the X-ray at $30^{\circ}$ in the lateral oblique position, we can pass through this facet in "tunnel vision". Entering through the facet joint permits entry into the foramen, making the technique successful.

This approach through the joint is closer to the limits of the Kambin triangle approach than it is to the classic subpedicular approach. Although the latter approach appears to deposit a higher concentration of medication in the anterior space than what is possible via the Kambin technique or the transfacetary technique, according to published studies, the results obtained do not seem to be significantly different.

\section{Conclusion}

The transforaminal block through the zygapophyseal joint presents an alternative to other approaches, such as the Kambin triangle approach or the subpedicular approach, when these approaches cannot be performed due to the patient's pathological status, which can make these approaches very difficult to carry out because of the anatomical particularities present in patients with more advanced degenerative osteoarthritis. 


\section{Acknowledgements}

None.

\section{Conflict of interest}

David Abejón: Proctor of Cardiva2 (Cosman, Abbott in Spain), PRIM (Nevro in Spain), Medtronic Ibérica and Boston scientific. No more conflict of interest for the rest of authors.

\section{References}

1. Simon LS. Relieving pain in America: A blueprint for transforming prevention, care, education, and research. J Pain Palliat Care Pharmacother. 2012;26:197-8.

2. Darnall BD, Ziadni MS, Roy A, et al. Comparative Efficacy and Mechanisms of a Single-Session Pain Psychology Class in Chronic Low Back Pain: Study Protocol for a Randomized Controlled Trial. Trials. 2018;19(1):165.

3. Price DD. Psycological and neural mechanisms of the affective dimension of pain. Science. 2000;9(288):1769-1772.

4. Mathews M, Mazhuvanchery C, Rab F. Post laminectomy syndrome: Treatment outcomes from a pain rehabilitation program. J Pain. 2014;15(4):S116.

5. Kreiner DS, Hwang SW, Easa JE, et al. An evidence-based clinica guideline for the diagnosis and treatment of lumbar disc herniation with radiculopathy. Spine J. 2014;14(1):180-191.

6. Jongen JLM, Hans G, Benzon HT, et al. Neuropathic pain and pharmacological Treatment. Pain Practice. 2013;14(3):283-95.

7. Mathieson S, Chiro M., Maher CG, et al. Trial of Pregabalin for Acute and Chronic Sciatica. N Engl J Med. 2017;376:1111-20.

8. Fukui S, Ohseto K, Shiotani M, et al. Distribution of referred pain from the lumbar zygapophyseal joints and dorsal rami. Clin J Pain. 1997;13:303-7.

9. Grubb SA, Lipscomb HJ, Coonrad RW. Degenerative adult onset scoliosis. Spine. 1988;13(3):241-245.

10. Kantrowitz F, Robinson DR, McGuire MB, et al. Corticosteroids inhibit prostaglandin production by rheumatoid synovia. Nature 1975;258:737739 .
11. Johansson A, Hao J, Sjolund B. Local corticosteroid application blocks transmission in normal nociceptive C-fibres. Acta Anaesthesiol Scand. 1990;34:335-338.

12. Vad VB, Bhat AL, Lutz GE, et al. Transforaminal epidural steroid injections in lumbosacral radiculopathy: a prospective randomized study. Spine. 2002;27(1):11-16.

13. Sluijter ME. In: Radiofrequency part 1. A, review of radiofrequency procedures in lumbar region. Chapter 7 . The DRG and the segmental nerve. Flivopress. 2001:119-138.

14. Finnerup NB, Otto M, McQuay HJ, et al. Research papers Algorithm for neuropathic pain treatment: An evidence based proposal. Pain. 2005;118(3):289-305.

15. Everett CR, Shah RV, Sehgal N, et al. A Systematic Review of Diagnostic Utility of Selective Nerve Root Blocks. Pain Physician. 2005;8(2):225233.

16. Reig E, Abejón D, Contreras R, et al. manual of Interventional procedures for pain treatment. Vol. I Radiofrequency. Cuarto Escalon, 2004.

17. Chopra P, Smith HS, Deer TR, et al. Role of adhenolysis in the management of chronic spinal pain: a systematic review of effectiveness and complications. Pain Physician. 2005;8(1):87-100.

18. Noth RB, Guarino AH. Spinal cord stimulation for failed back surgery syndrome: technical advances, patient selection and outcome. Neuromodulation. 1999;2(3):171-178.

19. Nelemans PJ, deBie RA, deVet HC, et al. Injection therapy for subacute and chronic benign low back pain. Spine. 2001;26:501-515.

20. Van Zundert J, Patijn J, Kessels A, et al. Pulsed radiofrequency adjacent to the cervical dorsal root ganglion in chronic cervical radicular pain: A double blind sham controlled randomized clinical trial. Pain. 2007;127(12):173-182.

21. Jan van $Z M D$, Maarten van KMD. Low Back Pain: From Algorithm to Cost-Effectiveness?. Pain Practice. 2005;5(3):179-189.

22. Kambin P, Sampson S. Posterolateral percutaneous suction-excision of herniated lumbar intervertebral discs. Report of interim results. Clin Orthop Relat Res. 1986;207:37-43. 